\title{
Aqueous Medium Preparation of Dialkyldiselenides
}

\author{
Tapasi Manna \\ Anup Kumar Misra* \\ Division of Molecular Medicine, Bose Institute, P-1/12, C. I. T. \\ Scheme VII M, Kolkata 700054, India \\ akmisra69@gmail.com
}

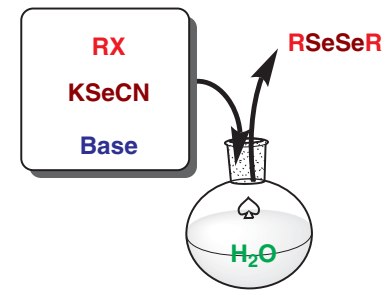

been prepared by the oxidation of selenols or selenoates. ${ }^{13}$ Diselenides have also been prepared through the formation a di-2-cyanoethyl diselenide derivative and its reaction with alkyl halides. ${ }^{14}$ In another approach, alkyl halide and aryl halide, ${ }^{15}$ diazonium ${ }^{16}$ or diaryliodonium salts, ${ }^{17}$ respectively, have been converted into alkyl or aryl selenocyanate derivatives with a variety of reagents. Treatment of selenocyanate derivatives with a base or reducing agent leads to the formation of selenols, which undergo aerial oxidation to furnish diselenide derivatives (Scheme 1). Diselenide derivatives have also been prepared using hydrogen selenide, produced by the treatment of elemental selenium with carbon monoxide and water. ${ }^{18}$ Despite their synthetic utilities, the reported methods for the preparation of diselenide derivatives suffer from several shortcomings, which include the use of strong reducing agents, toxic gasses, hazardous reaction conditions, poor yields and extended reaction times. Although, preparation of diselenide derivatives by the treatment of alkyl or aryl selenocyanates with hydroxides has been known for some time, ${ }^{19}$ the mechanistic aspects of this transformation have only recently been discussed. ${ }^{15}$ Therefore, it is pertinent to develop sustainable reaction conditions for the synthesis of diselenide derivatives avoiding hazardous reagents and solvents. ${ }^{20}$ Recently, reports have appeared describing the preparation of organoselenium derivatives using water as the reaction solvent. ${ }^{21}$ Soleiman-Beigi et al. reported ${ }^{22}$ the preparation of dialkyl diselenide derivatives by the reaction of alkyl halides and tosylates with elemental selenium in the presence of potassium hydroxide in water. In another report, Li et al. prepared $^{23}$ diaryl diselenides by copper-catalyzed coupling of aryl halides with elemental selenium in water (Scheme 2). 


\section{.}
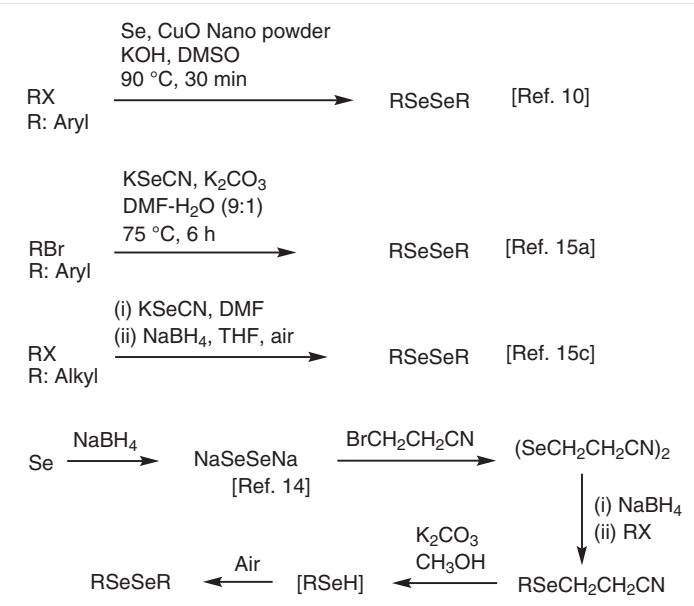

Scheme 1 Previously reported preparations of diselenides

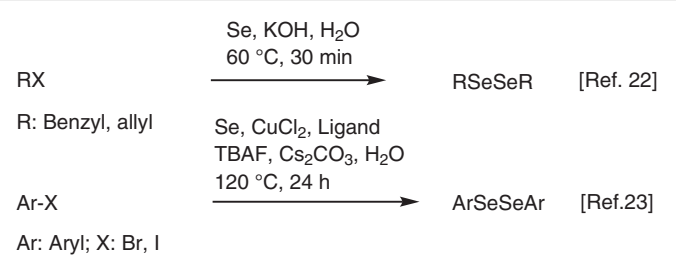

Scheme 2 Reported methods for the preparation of diselenide derivatives under aqueous reaction conditions

However, preparation of diselenide derivatives by the treatment of alkyl halides with potassium selenocyanate ( $\mathrm{KSeCN}$ ) followed by alkaline hydrolysis of the in situ generated alkyl selenocyanate in a one-pot, two-step reaction in water has remained unexplored to date. Water is an attractive solvent for several reasons such as cost effectiveness, safety, and being environmentally benign. ${ }^{24}$ In this context, a one-pot, two-step aqueous protocol is reported herein, involving treatment of alkyl halides with $\mathrm{KSeCN}$ followed by alkaline hydrolysis in water (Scheme 3 ).

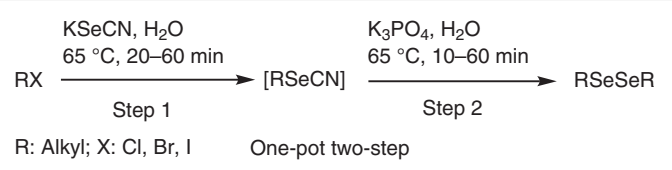

Scheme 3 Aqueous medium preparation of dialkyl diselenide derivatives from alkyl halide

In an initial set of experiments, benzyl bromide (1.0 mmol) was treated with $\mathrm{KSeCN}$, ranging from 1.0-2.0 equiv in water $(5 \mathrm{~mL})$ at a range of temperatures. It was observed that the use of 1.05 equiv of $\mathrm{KSeCN}$ in water $(5 \mathrm{~mL})$ at $65{ }^{\circ} \mathrm{C}$ resulted in the formation of benzyl selenocyanate in $90 \%$ yield in $30 \mathrm{~min}$. After formation of the selenocyanate derivative from benzyl bromide, a diverse number of bases such as $\mathrm{KOH}, \mathrm{K}_{2} \mathrm{CO}_{3}, \mathrm{~K}_{3} \mathrm{PO}_{4}, \mathrm{NaOH}, \mathrm{Na}_{2} \mathrm{CO}_{3}$, and $\mathrm{Et}_{3} \mathrm{~N}$ was added in excess to the reaction mixture in the same pot. It was observed that stirring the reaction mixture in the presence of $\mathrm{K}_{3} \mathrm{PO}_{4}$ at $65{ }^{\circ} \mathrm{C}$ furnished $90 \%$ dibenzyl diselenide in $30 \mathrm{~min}$. Use of $\mathrm{KOH}$ and $\mathrm{K}_{2} \mathrm{CO}_{3}$ also resulted in the formation of the diselenide derivative in a slightly lower yield. Use of $\mathrm{Et}_{3} \mathrm{~N}$ did not give the diselenide derivative, even after $24 \mathrm{~h}$ (Table 1). Therefore, $\mathrm{K}_{3} \mathrm{PO}_{4}$ was selected as the best choice for hydrolysis of the selenocyanate derivative. Use of a stoichiometric quantity of $\mathrm{K}_{3} \mathrm{PO}_{4}$ did not lead to complete diselenide formation even upon extended reaction times. However, use of an excess of $\mathrm{K}_{3} \mathrm{PO}_{4}$ ( 5 equiv) resulted in the formation of the diselenide derivative in a reasonably short reaction time.

Table 1 Optimization of Hydrolysis of In Situ Generated Selenocyanate Derivative (Step 2) using Different Bases ${ }^{\mathrm{a}}$

\begin{tabular}{lllll} 
& & & \\
\hline Entry & Base & Equiv & Time (min) & Yield (\%) \\
\hline 1 & $\mathrm{KOH}$ & 5 & 60 & 70 \\
2 & $\mathrm{NaOH}$ & 5 & 60 & 40 \\
3 & $\mathrm{~K}_{2} \mathrm{CO}_{3}$ & 5 & 45 & 40 \\
4 & $\mathrm{~K}_{3} \mathrm{PO}_{4}$ & 5 & 20 & 90 \\
5 & $\mathrm{~K}_{3} \mathrm{PO}_{4}$ & 2 & 120 & 75 \\
6 & $\mathrm{Na}_{2} \mathrm{CO}_{3}$ & 5 & 60 & 40 \\
7 & $\mathrm{Et}_{3} \mathrm{~N}_{2}$ & 10 & $24 \mathrm{~h}$ & - \\
\hline
\end{tabular}

${ }^{a}$ All reactions were carried out in water at $65^{\circ} \mathrm{C}$ after formation of the benzyl selenocyanate from benzyl bromide by treatment with KSeCN.

A wide variety of alkyl diselenide derivatives was prepared from the corresponding halide derivatives under similar reaction conditions (Table 2). However, in the case of aliphatic and aryloxyalkyl halides, the formation of selenocyanate derivatives was relatively slow, but reaction times were significantly reduced by adding tetrabutylammonium bromide (TBAB) $(0.1 \mathrm{mmol})$. In addition to simple alkyl halides, 6-deoxy-6-iodo-glycosides also furnished the corresponding diselenide derivatives under the optimized reaction conditions, although after extended reaction times. No trace of dialkyl selenide derivative was observed under these reaction conditions. ${ }^{25}$ 
Table 2 Preparation of Diselenide Derivatives by One-Pot, Two-Step Reaction in Water

\begin{tabular}{|c|c|c|c|c|}
\hline Entry & Starting material & Time $(\min )^{\mathrm{a}}$ & Product & Yield (\%) \\
\hline 1 & $1 a$ & $\begin{array}{l}10^{\mathrm{b}} \\
(30)^{\mathrm{b}}\end{array}$ & $2 a^{8 a}$ & 70 \\
\hline 2 & $1 b$ & $\begin{array}{l}10^{\mathrm{b}} \\
(30)^{\mathrm{b}}\end{array}$ & $2 \mathbf{b}^{7}$ & 72 \\
\hline 3 & $1 c$ & $\begin{array}{l}15^{\mathrm{b}} \\
(10)^{\mathrm{b}, \mathrm{d}}\end{array}$ & $2 c^{13}$ & 78 \\
\hline 4 & 1d & $\begin{array}{l}20^{\mathrm{b}} \\
(20)^{\mathrm{b}, \mathrm{d}}\end{array}$ & $2 d^{5 d}$ & 76 \\
\hline 5 & $1 e$ & $\begin{array}{l}30^{c} \\
(30)^{c}\end{array}$ & $2 \mathbf{e}^{11}$ & 90 \\
\hline 6 & $\begin{array}{l}M e \\
\mathbf{1 f}\end{array}$ & $\begin{array}{l}240^{c} \\
(15)^{c}\end{array}$ & $2 \mathbf{f}^{11}$ & 86 \\
\hline 7 & $1 \mathrm{~g}$ & $\begin{array}{l}10^{c} \\
(60)^{c}\end{array}$ & $2 \mathbf{g}^{11}$ & 84 \\
\hline 8 & 1h & $\begin{array}{l}60^{c} \\
(45)^{c, d}\end{array}$ & $2 \mathrm{~h}$ & 86 \\
\hline 9 & $1 \mathbf{i}$ & $\begin{array}{l}40^{c} \\
(30)^{c, d}\end{array}$ & $2 \mathbf{i}$ & 90 \\
\hline 10 & $\mathbf{1 j}^{\mathrm{N}}$ & $\begin{array}{l}60^{c} \\
(60)^{c, d}\end{array}$ & $2 j$ & 88 \\
\hline 11 & $1 k$ & $\begin{array}{l}180^{c} \\
(150)^{c}\end{array}$ & $2 k$ & 90 \\
\hline
\end{tabular}


Table 2 (continued)

12<smiles>CC(CBr)Sc1ccccc1</smiles>

11

13

14

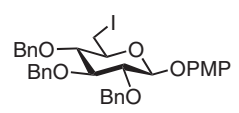

$1 \mathrm{~m}$
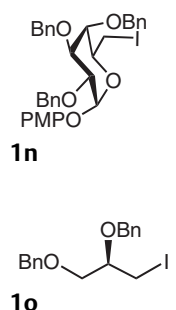

$10^{c}$

$(15)^{c, d}$

$(9 h)^{c, d}$

$60^{c}$

$(8 h)^{c, d}$

$15^{c}$

$(15)^{c}$<smiles>CC(C)Sc1ccccc1</smiles>

21

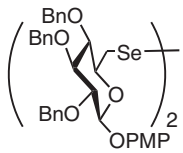

$2 m$

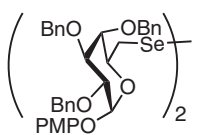

2n

$\left(\mathrm{BnO} \bigcup^{\mathrm{OBn}} \mathrm{Se}\right)$

20

\footnotetext{
a Time taken in step 1 is given in parentheses.

${ }^{\mathrm{b}}$ Reaction carried out at room temperature.

c Reaction carried out at $65^{\circ} \mathrm{C}$.

${ }^{\mathrm{d}}$ TBAB $(0.1 \mathrm{mmol})$ was added.
}

In summary, an efficient aqueous reaction protocol has been developed for the preparation of dialkyl diselenide derivatives from the corresponding alkyl halides by a one-pot, two-step reaction. ${ }^{26,27}$ The yields of the products are high. This reaction protocol has several advantages over previous procedures, such as operational simplicity, sustainability, short reaction times, high yields and simple work-up.

\section{Acknowledgment}

T.M. thanks UGC, New Delhi for providing a Junior Research Fellowship. The work is supported by the Science and Engineering Research Board (SERB), New Delhi (Project No. EMR/2015/000282 dated 17.09.2015) (AKM).

\section{References and Notes}

(1) (a) Devillanova, F. A. Handbook of Chalcogen Chemistry - New Perspectives in Sulfur, Selenium and Tellurium; Royal Society of Chemistry: Cambridge, 2007. (b) Wirth, T. Organoselenium Chemistry - Modern Developments in Organic Synthesis; Springer-Verlag: Heidelberg, 2000. (c) Derek, W. J.; Risto, L. Selenium and Tellurium Chemistry-From Small Molecules to Biomolecules and Materials; Springer-Verlag: Berlin, Heidelberg, 2011. (d) Oae, S. Organic Chemistry of Sulfur; Springer Science \& Business Media: Berlin, 2012.

(2) (a) Nogueira, C. W.; Zeni, G.; Rocha, J. B. T. Chem. Rev. 2004, 104, 6255. (b) Ibrahim, M.; Hassan, W.; Meinerz, D. F.; Dos Santos, M.; Klimaczewski, C. V.; Deobald, A. M.; Costa, M. S.; Noqueira, C. W.; Barbosa, N. B.; Rocha, J. B. Mol. Cell. Biochem. 2012, 371,
97. (c) Saito, G.; Swanson, J. A.; Lee, K.-D. Adv. Drug Delivery Rev. 2003, 55, 199. (d) Stefanello, S. T.; Prestes, A. S.; Ogunmoyole, T.; Salman, S. M.; Schwab, R. S.; Brender, C. R.; Dornelles, L.; Rocha, J. B. T.; Soares, F. A. A. Toxicol. in Vitro 2013, 27, 1433.

(3) (a) Mugesh, G.; duMont, W. W.; Sies, H. Chem. Rev. 2001, 101, 2125. (b) Geiger, P. G.; Lin, F.; Girotti, A. W. Free Radical Biol. Med. 1993, 14, 251. (c) Stadtman, T. C. Annu. Rev. Biochem. 1980, 49, 93. (d) Gucchait, A.; Joardar, N.; Parida, P. K.; Roy, P.; Mukherjee, N.; Dutta, A.; Yesuvadian, R.; SinhaBabu, S. P.; Jana, K.; Misra, A. K. Eur. J. Med. Chem. 2018, 143, 598. (e) Plano, D.; Baquedano, Y.; Moreno-Mateos, D.; Font, M.; Jiménez-Ruiz, A.; Palop, J. A.; Sanmartín, C. Eur. J. Med. Chem. 2011, 46, 3315.

(4) (a) Guy, R. G. In The Chemistry of Cyanates and their Thio- Derivatives, Part 2; Patai, S., Ed.; John Wiley \& Sons: New York, 1977, Chapter 18, p 819. (b) Erian, A. W.; Sherif, S. M. Tetrahedron 1999, 55, 7957.

(5) (a) Klayman, L. D.; Griffin, T. S. J. Am. Chem. Soc. 1973, 95, 197. (b) Lewicki, J. W.; Günther, W. H. H.; Chu, J. Y. C. J. Org. Chem. 1978, 43, 2672. (c) Krief, A.; Van Wemmel, T.; Redon, M.; Dumont, W.; Delmotte, C. Angew. Chem. Int. Ed. 1999, 38, 2245. (d) Doudin, K. I.; Berge, R. K.; Frøystein, N. Å.; Songstad, J. J. Chem. Soc., Perkin Trans. 1 2000, 723.

(6) (a) Gladysz, J. A.; Hornby, J. L.; Garbe, J. E. J. Org. Chem. 1978, 43, 1204. (b) Salama, P.; Bernard, C. Tetrahedron Lett. 1995, 36, 5711. (c) Syper, L.; Mlochowski, J. Tetrahedron 1988, 44, 6119.

(7) Li, J. Q.; Bao, W. L.; Lue, P.; Zhou, X.-J. Synth. Commun. 1991, 21, 799.

(8) (a) Krief, A.; Delmotte, C.; Dumont, W. Tetrahedron 1997, 53, 12147. (b) Krief, A.; Derock, M. Tetrahedron Lett. 2002, 43, 3083.

(9) Salama, P.; Bernard, C. Tetrahedron Lett. 1998, 39, 745.

(10) Singh, D.; Deobald, A. M.; Camargo, L. R. S.; Tabarelli, G.; Rodrigues, O. E. D.; Braga, A. L. Org. Lett. 2010, 12, 3288.

(11) Tian, F.; Yu, Z.; Lu, S. J. Org. Chem. 2004, 69, 4520. 
(12) Wang, J.-X.; Cui, W.; Hu, Y. J. Chem. Soc., Perkin Trans. 1 1994, 2341.

(13) Krief, A.; De Mahieu, A. F.; Dumont, W.; Trabelsi, M. Synthesis 1988, 131.

(14) Logan, G.; Igunbor, C.; Chen, G.-X.; Davis, H.; Simon, A.; Salon, J.; Huang, Z. Synlett 2006, 1554.

(15) (a) Krief, A.; Dumont, W.; Delmotte, C. Angew. Chem. Int. Ed. 2000, 39, 1669. (b) Mülar, J.; Terfort, A. Inorg. Chim. Acta 2006, 359, 4821. (c) Heredia, A. A.; Peñéñory, A. B. RSC Adv. 2015, 5, 105699. (d) Heredia, A. A.; Peñéñory, A. B. Beilstein J. Org. Chem. 2017, 13, 910

(16) Yavuz, S.; Disli, A.; Yildirir, Y.; Türker, L. Molecules 2005, 10, 1000.

(17) (a) Guan, Y.; Townsend, S. D. Org. Lett. 2017, 19, 5252. (b) Prabhu, K.; Chandrasekaran, S. Chem. Commun. 1997, 1021.

(18) Nishiyama, Y.; Hamanaka, S.; Ogawa, A.; Murai, S.; Sonoda, N. Synth. Commun. 1986, 16, 1059.

(19) Baker, J. W.; Moffitt, W. G. J. Chem. Soc. 1930, 1722.

(20) Anastas, P. T.; Warner, J. C. Green Chemistry: Theory and Practice; Oxford University Press: New York, 1998.

(21) (a) Freudendahl, D. M.; Santoro, S.; Shahzad, S. A.; Santi, C.; Wirth, T. Angew. Chem. Int. Ed. 2009, 48, 8409. (b) Santi, C.; Jacob, R. G.; Monti, B.; Bagnoli, L.; Sancineto, L.; Lenardão, E. J. Molecules 2016, 21, 1482.

(22) Soleiman-Beigi, M.; Yavari, I.; Sadeghizadeh, F. Phosphorus, Sulfur Silicon Relat. Elem. 2018, 193, 41.

(23) Li, Z.; Ke, F.; Deng, H.; Xu, H.; Xiang, H.; Zhou, X. Org. Biomol. Chem. 2013, 11, 2943.

(24) (a) Chanda, A.; Fokin, V. V. Chem. Rev. 2009, 109, 725. (b) Sarma, B. K.; Mugesh, G. Chem. Eur. J. 2008, 14, 10603. (c) Li, C.-J. Chem. Rev. 2005, 105, 3095. (d) Hailes, H. C. Org. Process Res. Dev. 2007, 11, 114. (e) Dallinger, D.; Kappe, C. O. Chem. Rev. 2007, 107, 2563.

(25) General method for the preparation of dialkyl diselenides: To a solution of alkyl halide $(1.0 \mathrm{mmol})$ in $\mathrm{H}_{2} \mathrm{O}(5 \mathrm{~mL})$ were added TBAB $(0.1 \mathrm{mmol})$ and $\mathrm{KSeCN}(1.05 \mathrm{mmol})$ and the reaction mixture was stirred vigorously at $65^{\circ} \mathrm{C}$ for the time detailed in Table $1 . \mathrm{K}_{3} \mathrm{PO}_{4}(5.0 \mathrm{mmol})$ was then added and the mixture was stirred at $65{ }^{\circ} \mathrm{C}$ for the time detailed in Table 2 . The reaction mixture was cooled and extracted with EtOAc $(2 \times 25$ $\mathrm{mL}$ ), and the organic layer was dried $\left(\mathrm{Na}_{2} \mathrm{SO}_{4}\right)$, filtered and concentrated. Chromatographic purification of the crude product over $\mathrm{SiO}_{2}$ furnished pure products. Analytical data of known compounds match with the data reported in the literature.

(26) Analytical data of novel compounds:

Di-(2-phenoxyethyl) diselenide (2h): Yellow oil; ${ }^{1} \mathrm{H}$ NMR (500 $\left.\mathrm{MHz}, \mathrm{CDCl}_{3}\right): \delta=7.29-7.21(\mathrm{~m}, 4 \mathrm{H}, \mathrm{Ar}-\mathrm{H}), 6.96-6.87(\mathrm{~m}, 6 \mathrm{H}$, Ar-H), $4.22\left(\mathrm{t}, \mathrm{J}=7.0 \mathrm{~Hz}, 4 \mathrm{H}, \mathrm{OCH}_{2}\right), 3.28(\mathrm{t}, \mathrm{J}=7.0 \mathrm{~Hz}, 4 \mathrm{H}$, $\left.\mathrm{SeCH}_{2}\right) ;{ }^{13} \mathrm{C}$ NMR $\left(125 \mathrm{~Hz}, \mathrm{CDCl}_{3}\right): \delta=157.2-113.6(\mathrm{Ar}-\mathrm{C}), 66.6$ (2 C), 27.1 (2 C); ESI-MS: $\mathrm{m} / \mathrm{z}=402.9[\mathrm{M}+\mathrm{H}]^{+}$; Anal. Calcd. for $\mathrm{C}_{16} \mathrm{H}_{18} \mathrm{O}_{2} \mathrm{Se}_{2}$ (401.96): C, 48.01; $\mathrm{H}, 4.53$; found: $\mathrm{C}, 47.84 ; \mathrm{H}, 4.75$. Di-(2-(4-methoxyphenoxy)ethyl) diselenide (2i): Yellow oil; ${ }^{1} \mathrm{H}$ NMR $\left(500 \mathrm{MHz}, \mathrm{CDCl}_{3}\right): \delta=6.86-6.74(\mathrm{~m}, 8 \mathrm{H}, \mathrm{Ar}-\mathrm{H}), 4.20(\mathrm{t}$, $\left.\mathrm{J}=7.0 \mathrm{~Hz}, 4 \mathrm{H}, \mathrm{OCH}_{2}\right), 3.76\left(\mathrm{~s}, 6 \mathrm{H}, \mathrm{OCH}_{3}\right), 3.25(\mathrm{t}, \mathrm{J}=7.0 \mathrm{~Hz}, 4 \mathrm{H}$, $\left.\mathrm{SeCH}_{2}\right) ;{ }^{13} \mathrm{C}$ NMR $\left(125 \mathrm{~Hz}, \mathrm{CDCl}_{3}\right): \delta=153.0-113.6(\mathrm{Ar}-\mathrm{C}), 67.4$ (2 C), 54.5 (2 C, $\left.\mathrm{OCH}_{3}\right), 27.2$ (2 C); ESI-MS: $\mathrm{m} / \mathrm{z}=462.9[\mathrm{M}+\mathrm{H}]^{+}$; Anal. Calcd. for $\mathrm{C}_{18} \mathrm{H}_{22} \mathrm{O}_{4} \mathrm{Se}_{2}$ (461.98): C, 46.97; $\mathrm{H}, 4.82$; found: $\mathrm{C}$, 46.80; $\mathrm{H}, 5.00$.
Di-(2-(4-nitrophenoxy)ethyl) diselenide (2j): Yellow oil; ${ }^{1} \mathrm{H}$ NMR (500 MHz, $\left.\mathrm{CDCl}_{3}\right)$ : $\delta=8.22-8.17(\mathrm{~m}, 4 \mathrm{H}, \mathrm{Ar}-\mathrm{H}), 6.98-6.92$ $(\mathrm{m}, 4 \mathrm{H}, \mathrm{Ar}-\mathrm{H}), 4.33\left(\mathrm{t}, \mathrm{J}=7.0 \mathrm{~Hz}, 4 \mathrm{H}, \mathrm{OCH}_{2}\right), 3.30(\mathrm{t}, \mathrm{J}=7.0 \mathrm{~Hz}$, $\left.4 \mathrm{H}, \mathrm{SeCH}_{2}\right) ;{ }^{13} \mathrm{C}$ NMR $\left(125 \mathrm{~Hz}, \mathrm{CDCl}_{3}\right): \delta=162.0-113.4(\mathrm{Ar}-\mathrm{C})$, 67.3 (2 C), 26.2 (2 C); ESI-MS: $\mathrm{m} / \mathrm{z}=492.9[\mathrm{M}+\mathrm{H}]^{+}$; Anal. Calcd. for $\mathrm{C}_{16} \mathrm{H}_{16} \mathrm{~N}_{2} \mathrm{O}_{6} \mathrm{Se}_{2}$ (491.93): C, 39.20; $\mathrm{H}, 3.29$; found: $\mathrm{C}, 39.00 ; \mathrm{H}$, 3.50 .

Di-(2-(2-naphthalenyloxy)ethyl) diselenide (2k): Yellow oil; ${ }^{1} \mathrm{H}$ NMR $\left(500 \mathrm{MHz}, \mathrm{CDCl}_{3}\right): \delta=7.78-7.63(\mathrm{~m}, 6 \mathrm{H}, \mathrm{Ar}-\mathrm{H}), 7.42-$ 7.37 (m, 2 H, Ar-H), 7.32-7.30 (m, 2 H, Ar-H), 7.14-7.08 (m, 4 H, $\operatorname{Ar}-\mathrm{H}), 4.38\left(\mathrm{t}, \mathrm{J}=7.0 \mathrm{~Hz}, 4 \mathrm{H}, \mathrm{OCH}_{2}\right), 3.36-3.31(\mathrm{t}, \mathrm{J}=7.0 \mathrm{~Hz}, 4 \mathrm{H}$, $\left.\mathrm{SeCH}_{2}\right) ;{ }^{13} \mathrm{C}$ NMR $\left(125 \mathrm{~Hz}, \mathrm{CDCl}_{3}\right): \delta=155.2-105.9(\mathrm{Ar}-\mathrm{C}), 66.7$ (2 C), 27.0 (2 C); ESI-MS: $\mathrm{m} / \mathrm{z}=02.9$ [M+H]+; Anal. Calcd. for $\mathrm{C}_{24} \mathrm{H}_{22} \mathrm{O}_{2} \mathrm{Se}_{2}$ (501.99): C, 57.61; H, 4.43; found: C, 57.45; H, 4.60. Di-(4-(phenylthio)butyl) diselenide (21): Yellow oil; ${ }^{1} \mathrm{H}$ NMR $\left(500 \mathrm{MHz}, \mathrm{CDCl}_{3}\right): \delta=7.31-7.13(\mathrm{~m}, 10 \mathrm{H}, \mathrm{Ar}-\mathrm{H}), 2.91(\mathrm{t}, \mathrm{J}=$ $7.0 \mathrm{~Hz}, 4 \mathrm{H}), 2.89(\mathrm{t}, \mathrm{J}=7.0 \mathrm{~Hz}, 4 \mathrm{H}), 1.90-1.80(\mathrm{~m}, 4 \mathrm{H}), 1.79-$ $1.71(\mathrm{~m}, 4 \mathrm{H}) ;{ }^{13} \mathrm{C}$ NMR $\left(125 \mathrm{~Hz}, \mathrm{CDCl}_{3}\right): \delta=135.5-124.8(\mathrm{Ar}-\mathrm{C})$, 32.1 (2 C), 28.8 (2 C), 28.1 (2 C), 27.8 (2 C); ESI-MS: m/z = 490.9 $[\mathrm{M}+\mathrm{H}]^{+}$; Anal. Calcd. for $\mathrm{C}_{20} \mathrm{H}_{26} \mathrm{~S}_{2} \mathrm{Se}_{2}$ (488.47): C, 49.18; H, 5.37; found: C, 49.00; H, 5.58.

(27) Bis-(p-methoxyphenyl 2,3,4-tri-0-benzyl-p-D-glucopyranosyl)-(6,6')-diselenide (2m): Yellow oil; ${ }^{1} \mathrm{H}$ NMR $(500 \mathrm{MHz}$, $\left.\mathrm{CDCl}_{3}\right): \delta=7.32-7.19(\mathrm{~m}, 30 \mathrm{H}, \mathrm{Ar}-\mathrm{H}), 7.01$ (d, J $=9.0 \mathrm{~Hz}, 4 \mathrm{H}, \mathrm{Ar}-$ H), 6.77 (d, J = 9.0 Hz, 4 H, Ar-H), 5.04 (d, J = 11.0 Hz, 2 H, PhCH), $4.92(\mathrm{~d}, \mathrm{~J}=11.0 \mathrm{~Hz}, 2 \mathrm{H}, \mathrm{PhCH}), 4.84(\mathrm{~d}, \mathrm{~J}=11.0 \mathrm{~Hz}, 2 \mathrm{H}, \mathrm{PhCH})$, $4.78(\mathrm{~d}, \mathrm{~J}=11.0 \mathrm{~Hz}, 2 \mathrm{H}, \mathrm{PhCH}), 4.75(\mathrm{~d}, \mathrm{~J}=7.5 \mathrm{~Hz}, 2 \mathrm{H}, \mathrm{H}-1, \mathrm{H}-$ $\left.1^{\prime}\right), 4.73(\mathrm{~d}, \mathrm{~J}=11.0 \mathrm{~Hz}, 2 \mathrm{H}, \mathrm{PhCH}), 4.58(\mathrm{~d}, \mathrm{~J}=11.0 \mathrm{~Hz}, 2 \mathrm{H}$, $\mathrm{PhCH}), 3.74\left(\mathrm{~s}, 6 \mathrm{H}, \mathrm{OCH}_{3}\right), 3.68-3.64(\mathrm{~m}, 4 \mathrm{H}, \mathrm{H}-2, \mathrm{H}-2$ ', $\mathrm{H}-3, \mathrm{H}-$ 3'), 3.62-3.57 (m, $\left.2 \mathrm{H}, \mathrm{H}-5, \mathrm{H}-5^{\prime}\right), 3.42-3.38$ ( $\mathrm{m}, 2 \mathrm{H}, \mathrm{H}-4, \mathrm{H}-4^{\prime}$ ), 3.37-3.34 (m, 2 H, H-6a, H-6'a), 3.12-3.06 (m, 2 H, H-6b, H6 'b); ${ }^{13} \mathrm{C} \mathrm{NMR}\left(125 \mathrm{~Hz}, \mathrm{CDCl}_{3}\right): \delta=155.37-114.5$ (Ar-C), $102.8(2$ C, C-1, C-1'), $84.4(2 \mathrm{C}), 82.2(2 \mathrm{C}), 80.8(2 \mathrm{C}), 75.7\left(2 \mathrm{C}, \mathrm{PhCH}_{2}\right)$, $75.2(2 \mathrm{C}), 75.0\left(2 \mathrm{C}, \mathrm{PhCH}_{2}\right), 74.9\left(2 \mathrm{C}, \mathrm{PhCH}_{2}\right), 55.5\left(2 \mathrm{C}, \mathrm{OCH}_{3}\right)$, 33.3 (2 C, C-6, C-6'); ESI-MS: $\mathrm{m} / \mathrm{z}=261.3[\mathrm{M}+\mathrm{Na}]^{+}$; Anal. Calcd. for $\mathrm{C}_{68} \mathrm{H}_{70} \mathrm{O}_{12} \mathrm{Se}_{2}$ (1238.31): C, 66.01; $\mathrm{H}, 5.70$; found: $\mathrm{C}, 65.82 ; \mathrm{H}$, 5.54 .

Bis-(p-methoxyphenyl 2,3,4-tri-0-benzyl- $\beta$-D-galactopyranosyl)-(6,6')-diselenide (2n): Yellow oil; ${ }^{1} \mathrm{H}$ NMR $(500 \mathrm{MHz}$, $\left.\mathrm{CDCl}_{3}\right): \delta=7.40-7.22(\mathrm{~m}, 30 \mathrm{H}, \mathrm{Ar}-\mathrm{H}), 7.06-7.04(\mathrm{~m}, 4 \mathrm{H}, \mathrm{Ar}-\mathrm{H})$, 6.77-6.74 (m, $4 \mathrm{H}, \mathrm{Ar}-\mathrm{H}), 5.27$ (d, J = 3.0 Hz, $\left.2 \mathrm{H}, \mathrm{H}-1, \mathrm{H}-1^{\prime}\right)$, 4.93-4.47 (m, $\left.12 \mathrm{H}, 6 \mathrm{PhCH}_{2}\right), 4.13-4.04\left(\mathrm{~m}, 4 \mathrm{H}, \mathrm{H}-2, \mathrm{H}-2^{\prime}\right.$ and H-3, H-3'), 4.00-3.96 (m, 2 H, H-5, H-5'), 3.70 (br s, $6 \mathrm{H}, 2 \mathrm{OCH}_{3}$ ), 3.66 (br s, 2 H, H-4, H-4'), 3.07-3.03 (m, 2 H, H-6a, H-6'a), 2.722.68 (m, $2 \mathrm{H}, \mathrm{H}-6 \mathrm{~b}, \mathrm{H}-6 \mathrm{\prime} \mathrm{b}) ;{ }^{13} \mathrm{C}$ NMR $\left(125 \mathrm{~Hz}, \mathrm{CDCl}_{3}\right)$ : $\delta=155.2-$ 114.5 (Ar-C), 98.3 (2 C, C-1, C-1'), 79.1 (2 C), 76.4 (2 C), 76.0 (2 C), $74.9(2 \mathrm{C}), 73.6(2 \mathrm{C}), 73.2(2 \mathrm{C}), 71.2(2 \mathrm{C}), 55.4\left(2 \mathrm{C}, \mathrm{OCH}_{3}\right)$, 30.6 (2 C, C-6, C-6'); ESI-MS: $\mathrm{m} / \mathrm{z}=261.3[\mathrm{M}+\mathrm{Na}]^{+}$; Anal. Calcd. for $\mathrm{C}_{68} \mathrm{H}_{70} \mathrm{O}_{12} \mathrm{Se}_{2}$ (1238.31): C, 66.01; $\mathrm{H}, 5.70$; found: $\mathrm{C}, 65.80 ; \mathrm{H}$, 5.55 .

Bis-(2,3-di-0-benzyloxy-(R)-propyl) diselenide (20): Yellow oil; ${ }^{1} \mathrm{H}$ NMR $\left(500 \mathrm{MHz}, \mathrm{CDCl}_{3}\right): \delta=7.34-7.22(\mathrm{~m}, 20 \mathrm{H}, \mathrm{Ar}-\mathrm{H})$, 4.63-4.48 (m, $\left.8 \mathrm{H}, \mathrm{PhCH}_{2}\right), 3.81-3.77(\mathrm{~m}, 2 \mathrm{H}), 3.62-3.56(\mathrm{~m}$, $4 \mathrm{H}), 3.19-3.15(\mathrm{~m}, 4 \mathrm{H}) ;{ }^{13} \mathrm{C}$ NMR $\left(125 \mathrm{~Hz}, \mathrm{CDCl}_{3}\right): \delta=138.2-$ 127.6 (Ar-C), 78.0 (2 C), $73.4(2 \mathrm{C}), 72.0(2 \mathrm{C}), 71.3(2 \mathrm{C}), 32.4(2$ C), 29.7 (2 C); ESI-MS: $\mathrm{m} / \mathrm{z}=71.1[\mathrm{M}+\mathrm{H}]^{+}$; Anal. Calcd. for $\mathrm{C}_{34} \mathrm{H}_{38} \mathrm{O}_{4} \mathrm{Se}_{2}$ (670.11): C, 61.08; H, 5.73; found: C, 60.90; $\mathrm{H}, 5.95$. 